\title{
Questes
}

\section{Rutebeuf ou la fabrique d'une poésie de résistance dans la crise universitaire (1254 - 1259)}

\section{Sung - Wook Moon}

\section{(2) OpenEdition}

\section{Journals}

\section{Édition électronique}

URL : http://journals.openedition.org/questes/5005

DOI : 10.4000/questes.5005

ISSN : 2109-9472

Éditeur

Les Amis de Questes

\section{Édition imprimée}

Date de publication : 13 juillet 2018

ISSN : 2102-7188

\section{Référence électronique}

Sung - Wook Moon, « Rutebeuf ou la fabrique d'une poésie de résistance dans la crise universitaire (1254 - 1259) », Questes [En ligne], 39 | 2018, mis en ligne le 30 juillet 2018, consulté le 21 décembre 2020. URL : http://journals.openedition.org/questes/5005; DOI : https://doi.org/10.4000/questes. 5005

Ce document a été généré automatiquement le 21 décembre 2020.

(c) Association des amis de «Questes » 


\title{
Rutebeuf ou la fabrique d'une poésie de résistance dans la crise universitaire (1254 - 1259)
}

\author{
Sung - Wook Moon
}

Dans les années 1250, Paris bouillonne de l'affrontement entre l'Université et les Ordres Mendiants ${ }^{1}$. C'est dans ce contexte que se réalise sous la plume de Rutebeuf une " première rencontre véritable [...] de la poésie et de l'histoire ${ }^{2}$ ». En soi, son engagement s'avouera être un échec. Les maîtres séculiers pour lesquels le rimeur a pris parti finiront par succomber devant les Frères qui, eux, seront soutenus par le Saint-Siège et la royauté capétienne. Qui plus est, à en juger par les textes conservés, Rutebeuf n'est intervenu dans le conflit universitaire que sur le tard. La querelle avait été déclenchée dès le début des années 1250, tandis que la plus ancienne des pièces du poète sur le sujet est de 1254. Le reste de sa production viendra après 1257 , lorsque la défaite des séculiers aura été scellée par l'exil de leur chef de file, Guillaume de Saint-Amour. Cette conjoncture entraîne des conséquences sur le travail d'écrire de Rutebeuf. Plus précisément, de la position inconfortable qu'elle lui assigne résultent la personnalité et la subjectivité dont il revêt son je poétique. Car si la «subjectivité littéraire » consiste en «ce qui marque le texte comme le point de vue d'une conscience ${ }^{3}$ ", la situation de celui qui résiste au courant du monde doit être propice à aiguiser la singularité de son point de vue $\mathrm{e}^{4}$.

Pour approfondir cette problématique, la présente étude se focalise sur les années 1254$1259^{5}$. Même si le combat de Rutebeuf continue jusqu'au début de la décennie suivante, la production précoce a son propre intérêt, surtout en ce qu'elle témoigne du tâtonnement dans la construction du personnage du poète résistant. À cet égard, nous relèverons d'abord des caractéristiques générales de la période en question. Ensuite, nous nous pencherons sur un exemple : la Complainte de Guillaume de Saint-Amour. Enfin, au moment de la conclusion, il sera question de clarifier les rapports intrinsèques qu'a l'invention d'une auctorialité avec celle d'une œuvre de résistance. 


\section{Question de terminologie : polémique, satire, pamphlet}

2 La position de Rutebeuf dans la querelle universitaire n'est pas à déterminer par la seule référence à son parti d'adhésion. Il faut considérer en termes globaux la configuration du champ discursif qu'une réflexion terminologique nous aide à éclairer.

3 Les critiques se sont servis de divers termes pour désigner la poésie universitaire de Rutebeuf, sans les distinguer explicitement les uns des autres. Le plus fréquent d'entre eux est celui de polémique. Le choix s'explique aisément, tant l'écrivain s'est engagé au sein d'une polémique. Il convient toutefois de remettre en question cette intériorité présumée. Si l'on suit Marc Angenot, selon lequel «le discours polémique suppose un milieu topique, un terrain commun aux entreparleurs ${ }^{6} »$, force est de constater que Rutebeuf n'a pas pu vraiment avoir accès à ce terrain. Le débat de l'Université est saturé d'autres langues que la sienne : traités, sermons, disputations, strictement encadrés par un protocole ritualisé, et toujours en latin?

4 Rutebeuf, pour sa part, n'écrit que des vers en langue vernaculaire. Bien qu'il promeuve ainsi la cause de son parti auprès de la masse urbaine, son œuvre doit apparaître comme vulgaire au regard des discours authentiquement universitaires. Dans ce sens, Rutebeuf est a priori exclu de la polémique.

5 Marc Angenot prend en considération deux autres vocables. Le premier, satire, présuppose un "monopole du bon sens" que l'auteur partage avec son lecteur. Sur la base de cette complicité se déploie " une rhétorique de mépris ${ }^{8}$ " par laquelle le satiriste ridiculise son adversaire comme absurde. De la même manière, la première des pièces universitaires de Rutebeuf assure que personne ne peut se tromper sur la fausseté des Jacobins :

Se par l'abit sont net et monde, Vous savez bien, ce est la voire, S'uns leus avoit chape roonde Si resambleroit il provoire ${ }^{9}$.

6 Malheureusement, ce qui est évident pour lui ne l'est pas pour tous ; «la voire ", à laquelle il croit, sera aussitôt foulée par la réalité. Alors, au lieu d'admettre son erreur et de baisser les bras, Rutebeuf atteste une crise des valeurs : l'imposture l'emporterait sur la vérité. Rien n'affaiblit donc sa conviction, quoique, les menteurs ayant accaparé le nom du vrai, ce soit dorénavant sa parole qui risque d'être traitée comme fausse et de s'exposer à l'oppression. Non seulement les adversaires déclarés le tourmentent, mais il est aussi affligé par ses prétendus amis qui restent indifférents et dont certains vont jusqu'à trahir la justice sans vergogne. Ainsi, en proportion avec l'affaissement de son clan, l'écriture de Rutebeuf passe de la satire au pamphlet ${ }^{10}$.

Des yex dou cuer ne veons gote,

Ne que la taupe soz la mote.

Entendez me vous, ne vous, voir?

Ou se vient chacun se dote.

Ahi! Ahi ! fole gent tote

Qui n'osez connoistre le voir [... $]^{11}$.

7 Cependant, le satirico-polémiste ne perd jamais de vue l'autre rive, celle de la polémique ; il guette l'occasion d'y faire incursion ou de la renverser. Son Dit de Guillaume de Saint- 
Amour se construit à l'instar d'un discours juridique, relativement tempéré et bien argumenté $^{12}$. Le Dit des règles, sur le ton farouche, n'en imite pas moins le sermon scolastique dans sa structure ${ }^{13}$. Ainsi, au moyen de quelques formes discursives privilégiées, le polémiste non attitré tente une autopromotion. Il s'agit également de contester le mauvais fonctionnement de l'Université, qui ferait fi de sa vocation primitive, à savoir la défense de la vérité. Les membres de cette haute institution de la chrétienté tolèreraient désormais jusqu'au pire des mensonges, à savoir l'hérésie :

Vous devin, et vous discretistre,

Je vous jete fors de mon titre,

De mon titre devez fors estre,

Quant le cinqueime esvengelitre

Vost on fere mestre et menistre

De parler dou roi celestre ${ }^{14}$.

8 La prévarication des savants a laissé un vide ; Rutebeuf l'occupe au nom de la vérité. Au bout de cette logique, refuser à ceux-là l'entrée à son propre «titre " revient à leur fermer la porte du paradis, car, de son côté, «Diex [les] giete de son regitre ${ }^{15}$.». Cette assimilation téméraire de sa propre volonté à celle de l'instance transcendante ${ }^{16}$ reflète paradoxalement la situation délicate où il est toujours moins vraisemblable de trouver ici-bas un quelconque secours.

Toutefois, pour un écrivain en marge, cela peut être aussi une façon de se venger de la forclusion qu'il aurait subie du cercle des cuistres, arrogamment repliés sur eux-mêmes.

\section{Imitatio Guillielmi?}

Certes, une telle posture de solitude relève de la topique. La mythologie antique comme l'Écriture sainte présentent une légion de prophètes méprisés par le peuple. Reste à observer que cette façon de parler va à l'encontre d'une hypothèse contextuelle retenue par la plupart des commentaires sur Rutebeuf: son œuvre aurait été une œuvre de commande. Il devait donc y avoir des commanditaires, maitres séculiers en l'occurrence, si bien que le poète n'était jamais aussi seul qu'il le déclarait. Pas plus qu'il n'aurait eu à inventer ses arguments ; lire d'autres auteurs, notamment Guillaume de Saint-Amour, lui aurait fourni « un arsenal de références toutes prêtes ${ }^{17}$ ».

10 L'auteur du Tractatus brevis de periculis novissimorum temporum a une importance particulière pour Rutebeuf. C'est au moment de son exil, en 1257, que la plume du poète commence à avoir le vent en poupe. Le premier poème de cette phase est un Dit de Guillaume de Saint-Amour, avant une Complainte en l'honneur du même homme. De là, d'aucuns ont imaginé une intimité entre eux, comme celle qui lierait un élève à son professeur ${ }^{18}$.

11 À force de conjectures, pourtant, on a négligé une évidence : Rutebeuf prend Guillaume de Saint-Amour moins pour auteur que pour exemple. De fait, même quand il puise dans l'ouvrage du maître, le poète ne mentionne jamais sa source. Le cas-limite en est le dit $\mathrm{Du}$ Pharisien. Si son titre rappelle un sermon de Guillaume, De pharisaeo et publicano ${ }^{19}$, le fameux personnage biblique ne fait pas apparition dans le texte du poème. Pareillement, la conception diffère d'un côté à l'autre. Le sermon latin n'exploite qu'accessoirement l'une des thématiques favorites du prédicateur: le soupçon d'hérésie, facilité par la 
malencontreuse publication de l'Évangile éternel par le franciscain Gerardo de Borgo San Donnino ${ }^{20}$.

Ce soupçon, en revanche, se transforme en certitude dans le vers français, dont l'attaque dénonce un funeste cousinage entre Hypocrisie - emblème des Frères - et Hérésie :

Seignor qui Dieu devez amer [...]

A vous toz faz je ma clamor

D’Ypocrisie,

Cousine germaine Heresie,

Qui bien a la terre saisie ${ }^{21}$.

13 Cette association d'Hypocrisie et d'Hérésie provient de Guillaume, notamment de son De periculis. Le théologien a toutefois évité de l'imputer explicitement à ses ennemis réels, les Mendiants, et s'est borné à de vagues allusions, tandis que le poète n'hésite pas à actualiser ce qui restait en attente d'un "welcome misunderstanding ${ }^{22}$ ». De plus, Rutebeuf le transpose dans une vision élargie des « dangers des derniers temps » et des «menaces des temps actuels ${ }^{23}$ ", pour en conclure : «Que c'est contre l'avenement /A Antecrist ${ }^{24}$ ». Cette interprétation synthétique des thèses de Guillaume prouve que nous avons affaire à un lecteur attentif et intelligent ${ }^{25}$. Mais, en même temps, la figure d'autorité se trouve soigneusement éclipsée ${ }^{26}$ par celui qui fait mine d'avoir élaboré lui-même sa démonstration :

Or vous vueil dire de son estre,

Qui sont si seignor et si mestre

Parmi la vile.

Diex les devise en l'Evangile, Qui n'est de barat ne de guile,

Ainz est certaine :

Granz robes ont de simple laine

Et si sont de simple couvaine $[. . .]^{27}$.

Le portrait des hypocrites se donne comme mélange des observations que Rutebeuf aurait faites « parmi la vile» d'une part, et de ce que lui aurait enseigné «l'Evangile » d'autre part. Ainsi s'enchevêtrent savoirs et expériences, généralités et particularités, de la même manière que la figure allégorique d'Hypocrisie avait croisé, ici en France, des Frères individualisés par leurs prénoms :

Ypocrisie la renarde,

Qui defors oint et dedenz larde,

Vint ou roiaume.

Tost ot trové Frere Guillaume,

Frere Robert et Frere Aliaume,

Frere Giefroi,

Frere Lambert, Frere Lanfroi ${ }^{28}$.

15 Cette écriture ne se réduit pas à une mise en rime et mise en roman de la prose latine de Guillaume, pas plus qu'elle n'en est une simple adaptation imagée. Elle fait graviter tous les ingrédients transmis autour du nouveau point central qui lui est propre, le $j e^{29}$, dont il est dit :

S'en un mauvés leu ensailloie,

Ne peut el estre ${ }^{30}$. 
Plus que le titre de vulgarisateur d'un modèle, Rutebeuf revendique sa propre raison d'être. Se mettre à l'épreuve dans le "mauvés leu», s'en faire témoin ou martyr ${ }^{31}$, constituent son atout vis-à-vis des universitaires qui, tout en vivant au sein des milieux urbains, tendent à construire un univers clos et privilégié ${ }^{32}$. Ce faisant, certainement, Rutebeuf demeure fidèle à Guillaume, l'objectif de celui-ci étant d'aider les contemporains à reconnaître « les hypocrites de [leur] temps ${ }^{33}$ ». Mais pour bien mettre en œuvre cette théorie, le praticien la verse dans sa propre langue où son vécu individuel vaut mieux que tout argument et où sa présence personnelle sert de pierre de touche au réel.

\section{La Complainte de Guillaume : voix prêtée et parole usurpée}

Nous avons vu que, d'une part, l'écriture pamphlétaire tenait à la position inconfortable de l'écrivain et que, d'autre part, Rutebeuf cherchait à acquérir son propre statut par et dans ce qu'il a écrit. C'est de ce point de vue que nous allons analyser la Complainte de Guillaume. De prime abord, cet échantillon peut paraître inadéquat pour la simple raison que le je du poète y est pratiquement absent. Le texte consiste en une prosopopée où Église monopolise le discours, en déplorant les malheurs de Guillaume ainsi que les siens :

«Vous qui alez parmi la voie,

Arestez vous et chascuns voie

S'il est dolor tel com la moie",

Dist sainte Yglise ${ }^{34}$.

Selon Emmanuelle Poulain-Gautret, il s'agirait d' ' une variation et un approfondissement du motif du "parler pour l'autre" (à tous les sens de l'expression "parler pour") : le "je" narrateur est le porte-parole d'Église, qui elle-même parle pour Guillaume de SaintAmour ». Cela dit, il est hâtif d'en induire que ce je est « un simple outil qui ne renvoie pas à un "moi" » et que « le poète n'est pas une vraie personne ${ }^{35}$ ". C'est même l'inverse : sous prétexte de concéder sa voix, Rutebeuf tente plutôt d'instrumentaliser la parole d'autrui pour que celle-ci serve sa cause contestataire.

Examinons dans cette optique la structure énonciatrice du texte. La locutrice n'est nommée qu'au vers 4. Jusque-là, à supposer qu'il n'y ait pas de guillemets, comme sur le manuscrit médiéval ou lors d'une récitation publique, les trois premiers vers ont toute l'apparence d'un simple discours à la première personne. Mais ils traduisent en réalité un passage des Lamentations (I, 12) qui, très connu au Moyen Âge, ne devait pas passer inaperçu ${ }^{36}$. Ce brouillage soulève la question de savoir à qui revient le dire avant que le poète n'intervienne pour désigner Église. Avec ce geste fugitif, cependant, loin de s'effacer derrière la protagoniste identifiée, celui-ci affirme sa présence de deux manières. Le quatrième vers, le seul qui lui est réservé sur l'ensemble du poème, fait percevoir une particularité fondamentale de ce discours qui est un discours rapporté : l'écart entre le temps d'énoncé et le temps d'énonciation. Autrement dit, le propos passé d'Église ne nous est entendu que grâce à la voix de celui qui à présent le transmet et qui, tout en la nommant, se distingue de cette locutrice.

La présence de cet intermédiaire, indispensable pour le fonctionnement du texte, est davantage accentuée par la prosodie syncopée du tercet couée ${ }^{37}$. Cette singularité formelle ne se révèle pas dans les vers 1-3 constituant un tercet d'octosyllabes monorime, jusqu'à 
ce que le quatrième brise la monotonie, à la fois par le changement de mètre et de rime. Ainsi, sur ce quadrisyllabe d'allure anodine, se révèle la touche d'un poète ingénieux.

\section{De consolation à complainte}

Cette ingéniosité est à mettre en relation avec l'usage d'un modèle littéraire :

Tel gent se font de ma querele

Qui me metent en la berele :

Les miens ocient

Sanz ce que pas ne me desfient,

Ainz sont a moi, si comme il dient

Por miex confondre ${ }^{38}$.

Afin de dénoncer les Mendiants hypocrites, Rutebeuf s'inspire là d'une phrase de la Consolation de Philosophie : «Quae vero pestis efficiacior ad nocendum quam familiaris inimicus ${ }^{39}$ !». Mais se produit à nouveau un glissement. Chez Boèce, la maxime a été prononcée par Philosophie, laquelle entendait détourner son disciple de l'amitié mondaine et lui insuffler l'esprit de détachement. Par contre, la Complainte de Guillaume fait entendre un cri d'amertume, débordant de loin l'apathie philosophique. Ce cri est d'ailleurs sorti d'Église qui, pour personnifiée qu'elle soit, n'assume pas la même charge que son homologue de la Consolation. Elle apparaît même plus tourmentée et plus menacée que Guillaume de Saint-Amour, nouveau Boèce ${ }^{40}$.

La citation adaptée n'est pas la seule piste boétienne. Voici une deuxième :

Ha! Fortune, chose legiere,

Qui oins devant et poins derriere,

Comme es marrastre ${ }^{41}$ !

Si Fortune ${ }^{42}$ pesait lourdement sur la démarche de la Consolation, elle n'y a été introduite que pour être à jamais domptée et intégrée par la sage maîtresse de Boèce ${ }^{43}$. À l'inverse, Église ne réussit pas à brider cette force maléfique, elle qui, à un moment, laisse échapper un oracle peu rassurant :

Or [Guillaume] lera donc Fortune corre,

Qu'encontre li ne puet acorre,

C'est or la somme ${ }^{44}$.

Dans l'œuvre de Boèce, en effet, la consolation par Philosophie requérait «la mise entre parenthèses d'une composante essentielle de l'expérience humaine [...] le temps ${ }^{45}$ ». Cette belle astuce ne marche pas bien dans une complainte qui surgit avec une prise de conscience du temps. Tiraillée entre le passé regretté et l'avenir qui s'annonce mal, comme sous le choc de la soudaine déchéance de Guillaume, la complainte est régentée par Fortune $e^{46}$.

Alors se pose une question: à quoi sert la complainte; en quoi contribue-t-elle à un objectif dont sa teneur nie toute possibilité de réalisation? Miser sur la vengeance divine est envisageable, mais un tel ultimatum cache à peine la résignation au niveau humain ${ }^{47}$. Toujours est-il que la Complainte de Guillaume offre une ligne de conduite avec un exemple à suivre, à condition qu'on ne le cherche pas auprès du héros éponyme. Tout au long de la tirade d'Église, Guillaume est si bien maintenu en silence qu'il a l'air d'être déjà attiré vers un univers transcendantal ${ }^{48}$, apparaissant plus comme modèle à admirer que comme 
modèle à imiter. Le texte trace donc un deuxième modèle, cette fois-ci accessible. Ce modèle alternatif est doté d'une voix humaine, qui tremble, mais trouve le courage d'affronter les menaces. Donner à cette voix le nom d'Église, c'est une façon d'établir une communauté de douleur à l'image de celle-ci qui a été fondée sur la mémoire de la Passion. Dans le poème où la première personne du pluriel est grammaticalement absente, Église fonctionne comme un nous de substitution, un nous compatissant.

Pour autant, Église elle-même n'est qu'impersonnelle et abstraite tant qu'elle n'a pas recruté une personne, un premier adhérent autour duquel s'agrégera un collectif. En livrant sa voix à la parole d'Église, en lui donnant une sorte de substance, le poète remplit ce rôle déterminant. Mais cette parole, en retour, subit une modulation significative. Lorsqu'Église gémit que « n'est més nus qui le [ = Guillaume] ramentoive ${ }^{49}$ », il s'agit d'une prétérition, figure de style d'autocontradiction, puisque celle qui y parle, au moins elle, «ramentoi[t] » bien la victime supposément oubliée par tous ${ }^{50}$. Encore faut-il remarquer que cette contradiction n'est pas d'ordre purement rhétorique, mais corrélative à la structure du texte. Comme nous l'avons constaté, le discours rapporté implique un décalage temporel entre énoncé et énonciation. Cela installe un jeu qui peut aboutir à démentir ce qui est en train de se rapporter. Aussi, quand il transmet le constat passé d'Église, le poète-rapporteur se fait-il celui « qui ramentoi[t] » à présent à la fois l'exilé Guillaume et l'Église tourmentée. Cet acte de mémoire, qui est acte d'écrire, dépasse la contradiction rhétorique par la force d'une voix actuelle.

Ce tour de force fait penser à un aspect de l'engagement de Rutebeuf : il est arrivé trop tard dans la querelle. Mais c'est aussi un choix stratégique. Cette poésie d'après-coup qu'est la complainte présuppose un complaignant qui chancelle mais demeure debout. Son logos désespéré est chargé non seulement de pathos exagéré, mais aussi d'ethos de ténacité. Le désespoir n'interdit point une résistance. La lutte continue.

En fin de compte, l'ethos que nous venons de déceler de même que la personnalité qui le porte peuvent être estimés flous. On remarquera notamment l'absence d'un élément représentatif de cette personnalité : le nom. De fait, Rutebeuf s'est rarement nommé dans le corpus universitaire, et jamais dans les années 1254-1259. D'après Edmond Faral, ce serait à cause de la peur de persécution ${ }^{51}$ : le dissident, sous sa verve fougueuse, aurait-il donc tenu à sa peau ?

Nous émettons une autre hypothèse : le nom surgit à la suite d'une identité auctoriale que Rutebeuf n'a pas acquise depuis toujours, mais élaborée dans la durée de son parcours d'écriture. L'engagement universitaire y a joué le rôle d'accélérateur, sinon de déclencheur, en exigeant l'installation d'un je de résistant au sein de l'écriture de résistance. Le nom ne fera apparition dans le texte que comme nœud de cette quête d'identités ${ }^{52}$.

Effectivement, pendant la période anonyme d'avant 1260 , on trouve des poèmes où Rutebeuf se donne pleinement le statut d'auteur, comme celui-ci :

Puis qu'il covient verité tere,

De parler n'ai je més que fere.

Verité ai dite en mains leus :

Or est li dires pereilleus

A cels qui n'aiment veritées [...].

30 Le troisième vers renvoie à des compositions antérieures, censément connues du public comme ayant été écrites par Rutebeuf. Les textes individuels sont intégrés comme 
différentes facettes dans un continuum qui, en dernière analyse, n'est rien d'autre que la vie continue d'une personne, d'un écrivain. Ainsi, sans qu'il y ait le nom, une auctorialité est affirmée. D'autant plus clairement que cet auteur ne l'est devenu qu'en tant qu'auteur résistant, parce que dans la tradition occidentale, à en croire Michel Foucault, « les textes, les livres, les discours ont commencé à avoir réellement des auteurs [...] dans la mesure où l'auteur pouvait être puni, c'est-à-dire dans la mesure où les discours pouvaient être transgressif ${ }^{54} »$.

Si, pour les uns, il est un brave témoin de la vérité désertée, il n'est qu'un fomenteur de trouble pour les autres. Cette équivoque périlleuse est inhérente à la condition du résistant. Rutebeuf l'assume, lui qui avoue craindre "cels qui n'aiment verité », mais qu'aucune crainte n'empêchera de dire 182 vers véridiques. La menace ne fait que renchérir sur l'audace de celui qui dit, qui dit je. Fluctuat nec mergitur.

\section{NOTES}

1. Sur cette affaire, voir Michel-Marie Dufeil, Guillaume de Saint-Amour et la polémique universitaire parisienne. 1250 - 1259, Paris, Picard, 1972.

2. Paul Zumthor, Essai de poétique médiévale [1972], Paris, Éditions du Seuil, 2000, p. 487.

3. Michel Zink, La subjectivité littéraire. Autour du siècle de saint Louis, Paris, PUF, 1985, p. 8.

4. Nous poursuivons ainsi le chemin entrouvert par Nancy Freeman Regalado: « The encounter between poetry and history, which takes place in Rutebeuf's University poems, is perhaps the necessary first step toward our modern conception of poetry as an expression of an individual consciousness ", dans Poetic Patterns in Rutebeuf. A Study in Noncourtly Poetic Modes of the Thirteenth Century, New Haven/Londres, Yale University Press, 1970, p.188. C'est cette affirmation qui a conduit Paul Zumthor à énoncer la sienne, citée ci-dessus, en termes plus concis et «[non] sans exagération", compte tenu d'exemples plus anciens tels que Peire Cardenal (Nico Van den Boogaard, "La forme des polémiques et les formes poétiques: dits et motets du XIII siècle » [1976], dans Autour de 1300. Études de philologie et de littérature médiévales, Amsterdam, Rodopi, 1985, p. 19-40, cit. p. 20).

5. Années pendant lesquelles sont écrits les poèmes suivants, selon l'ordre de notre édition de référence : Discorde de l'Université et des Jacobins (1254); Dit de Guillaume de Saint-Amour (1257) ; Du Pharisien (1259); Complainte de Guillaume (1259); Des règles (1259); Dit de sainte Église (1259) (Euvres complètes de Rutebeuf, éd. Edmond Faral et Julia Bastin, Paris, Picard, 1959-1960, 2 vol., t.1, p. 238-285 : nous renverrons à cette édition par le sigle «F-B »). Michel-Marie Dufeil, suivi par Michel Zink, essaye de corriger la chronologie proposée par Edmond Faral et Julia Bastin (MichelMarie Dufeil, «L'œuvre d'une vie rythmée : chronographie de Rutebeuf », dans Musique, littérature et société au Moyen Âge, dir. Danielle Buschinger et André Crépin, Paris, Champion, 1980, p. $279-$ 294, en particulier p. 281-283 ; Rutebeuf, Euvres complètes [1989-1990], éd. et trad. Michel Zink, Paris, Librairie Générale Française, coll. «Lettres gothiques », 2001, p. 115-121 et 135-191). Mais les nouvelles hypothèses ne sont guère plus convaincantes que les anciennes, lesquelles sont suivies ici. Tous, au demeurant, sont d'accord pour mettre les six textes en question dans le créneau 1254-1259.

6. Marc Angenot, «La parole pamphlétaire. Esquisse d'un cadre typologique », Études littéraires, vol. 11, n², 1978, p. 255-264, cit. p. 260. 
7. À propos notamment de la pratique de la disputatio qui, en raison de ses exigences formelles, n'aurait pas pu aboutir à l'épanouissement d'un espace public au sens habermasien du terme, voir les deux études suivantes, publiées dans un même ouvrage collectif: Bénédicte Sère, "La disputatio dans l'université médiévale: esquisse d'un usage public du raisonnement?», dans L'espace public au Moyen Âge. Débats autour de Jürgen Habermas, dir. Patrick Boucheron et Nicolas Offenstadt, Paris, PUF, 2011, p. 251-262 ; Corinne Leveleux, «Controverses juridiques et désarmement herméneutique, ou la brève histoire d'un espace public doctrinal chez les juristes ( XII ${ }^{\mathrm{e}}$-XIII ${ }^{\mathrm{e}}$ siècles) », dans ibid. , p. 263-275.

8. Marc Angenot, « La parole pamphlétaire », art. cit., p. 260-261.

9. Rutebeuf, Discorde de l'Université et des Jacobins, v. 45-48, dans F-B, éd. cit., t. 1, p. 240. Le poète rappelle bien sûr le fameux proverbe:»Li abis ne fait pas le relegieus » (Proverbes français antérieurs au XV siècle, éd. Joseph Morawski, Paris, Champion, 1925, p. 39, n 1053).

10. Pour les traits généraux du pamphlet, Marc Angenot, «La parole pamphlétaire », art. cit., p. 261-262.

11. Rutebeuf, Dit de sainte Église, v.13-18, dans F-B, éd.cit., t.1, p. 279. Pour le vers 16, hypométrique, où il est envisageable de corriger "vient", soit en devient, soit en nient, voir Edmond Faral, « À propos de l'édition des textes anciens : le cas du manuscrit unique », dans Recueil de travaux offerts à M. Clovis Brunel, Paris, Société de l'École des chartes, 1955, p. 409-421, cit. p. 412-413.

12. Voir Nancy Freeman Regalado, Poetic Patterns in Rutebeuf, op. cit., p. 160-163 ; Béatrice Périgot, «Controverse, invective et vérité chez Rutebeuf », Camaren, vol. 1, 2006, p. 19-35, en particulier p. 26-29.

13. Voir Nico Van den Boogaard, «La forme des polémiques et les formes poétiques », art. cit., p. 34-40.

14. Rutebeuf, Dit de sainte Église, v. 37-42, dans F-B, éd. cit., t. 1, p. 280.

15. Ibid., v. 48.

16. Ce geste est préparé dès la première strophe par une adresse directe au Christ: «Ha! Jhesucriz, car te ravise /Que la lumiere soit esprise /C'on a estaint pour toi despire ! /La loi que tu nous as aprise /Est si vencue et entreprise /Qu'elle se torne a desconfire » (ibid., v. 7-12, dans F-B, éd. cit., t. 1, p. 279). Et juste avant ces vers, la révélation du ressort de son écriture - le cuer taint et plain d'ire» (ibid., v.5) - nous renvoie à une caractéristique du pamphlet: "L'énonciateur se signale dans le texte comme dépourvu de statut ou de mandat - mais automandaté par une conviction de for intérieur. La parole pamphlétaire n'a d'autre légitimité que celle qu'elle tire d'une vérité absente » (Marc Angenot, « La parole pamphlétaire », art. cit., p. 262).

17. F-B, éd. cit., t. 1, p. 37.

18. Michel-Marie Dufeil présume que Rutebeuf avait été disciple de Guillaume dans la Faculté des Arts de Paris (voir Guillaume de Saint-Amour, op.cit., p. 51). Et Denis Huë se demande si le professeur proscrit, en voyage secret à Paris, n'aurait pas pu dicter à son porte-parole les grandes lignes du Dit de Guillaume, tout en admettant que cette histoire imaginée « relève du feuilleton plus que de la critique littéraire » (Denis Hüe et Hélène Gallé, Rutebeuf, Neuilly, Atlande, 2006, p. 68). À l'opposé, Nancy Freeman Regalado veut remettre en doute la sincérité des opinions de Rutebeuf qui reproduiraient simplement celles de ses clients du moment (voir Poetic Patterns in Rutebeuf, op. cit., p. 72).

19. Le poème a d'autres intitulés dans les deux autres manuscrits que celui qui sert de base à notre édition de référence (Paris, BnF, français 837) : soit Autre dist d'Ypocrisie (Paris, BnF, français 1593), soit D’Ypocrisie (Paris, BnF, français 1635). Mais le titre Du Pharisien semble avoir plus de chance d'être authentique en tant que lectio difficilior. Quant au sermon de Guillaume, il est présenté et édité par Andrew Traver, The Opuscula of William of Saint-Amour. The Minor Works of 1255-1256, Münster, Aschendorff, 2003, p. $70-74$ et $191-205$. 
20. Voir ibid., p. 74. Sur l'Évangile éternel, compilation de textes eschatologiques de Joachim de Flore et dont l'Introduction exposa son auteur à la condamnation de la Curie, voir Michel-Marie Dufeil, Guillaume de Saint-Amour, op. cit., p. $124-127$.

21. Rutebeuf, Du Pharisien, v. 1 et 6 - 9, dans F-B, éd. cit., t. 1, p. $250-251$.

22. Sita Steckel, «Narratives of Resistance: Arguments against the Mendicants in the Works of Matthew Paris and William of Saint-Amour ", dans Thirteenth Century England, vol. 15, Authority and Resistance in the Age of Magna Carta, dir. Janet Burton et al., Mertlesham, The Boydell Press, 2015, p. 157-177, cit. p. 173.

23. Tel est le double sens du titre équivoque De periculis novissimorum temporum. Voir MichelMarie Dufeil, Guillaume de Saint-Amour, op. cit., p. 212.

24. Rutebeuf, Du Pharisien, v. 102-103, dans F-B, éd. cit., t. 1, p. 254.

25. Edmond Faral souligne que « ce jongleur était un homme instruit [...]. Il n'a pas pris la défense de Guillaume de Saint-Amour sans savoir ce qu'étaient ses idées » («Pour le commentaire de Rutebeuf. Le dit des "règles" ", Studi Medievali, vol. 16, 1943-1950, p. 176-211, cit. p. 206-207).

26. À une exception près : « N'est més nus tels qui la [ = Hypocrisie] responde /Que maintenant ne le confonde /Sanz jugement » (Rutebeuf, Du Pharisien, v. 98-100, dans F-B, éd. cit., t. 1, p. 254). On remarquera néanmoins que ce passage absorbe le rappel du bannissement de Guillaume dans le flou d'une généralité anonyme.

27. Rutebeuf, Du Pharisien, v. 45-52, dans F-B, éd. cit., t. 1, p. 252.

28. Ibid., v. 80-86, dans F-B, éd. cit., t. 1, p. 253.

29. Cela différencie le poète de Guillaume qui, dans son De periculis, se cache derrière la première personne au pluriel, en définissant son travail comme une collection de fragments scripturaires et en soulignant que rien n'y a été inventé. Voir De periculis novissimorum temporum, prologue, éd. et trad. Guy Geltner, Paris/Leuven/Dudley, Peeters, 2008, p. 44.

30. Rutebeuf, Du Pharisien, v. 43 - 44, dans F-B, éd. cit., t. 1, p. 252.

31. Le mot martyr signifie étymologiquement témoin. C'est pourquoi « on n'est pas martyr parce qu'on meurt, on meurt parce qu'on est martyr» (Victor Saxer, «Aspects de la typologie martyriale. Récits, portrait et personnages ", Publications de l'École française de Rome, t. 149, $\mathrm{n}^{\circ} 1$, 1991, p. 321 - 331, cit. p. 326). La fin surprenante du Dit de Guillaume se comprendrait en ce sens : «Endroit de moi vous puis je dire/Je ne redout pas le martire/De la mort, d'ou qu'ele me viegne, /S'ele me vient por tel besoingne » (v. 117 - 120, dans F-B, éd. cit., t. 1, p. 248).

32. Voir Jacques Verger, Les universités au Moyen Âge [1973], Paris, PUF, 2013, p. 76 - 78.

33. "Per praedictum Pharisaeum qui erat hypocrita [...] significantur hypocritae nostri temporis " (Guillaume de Saint-Amour, De pharisaeo et publicano, §5, dans Andrew Traver, The Opuscula of William of Saint-Amour, op.cit., p. 193 ; nous traduisons: «Par le susdit Pharisien qui était hypocrite [...] sont indiqués les hypocrites de notre temps »).

34. Rutebeuf, Complainte de Guillaume, v. 1-4, dans F-B, éd. cit., t. 1, p. 258.

35. Emmanuelle Poulain-Gautret, " "Mout a entre faire et dire". Rutebeuf et Guillaume de SaintAmour ", dans Romans d'Antiquité et littérature du Nord. Mélanges offerts à Aimé Petit, dir. Sarah Baudelle-Michels et al., Paris, Champion, 2007, p. 687 - 698, cit. p. 691, p. 693 et p. 695.

36. « $O$ vos omnes qui transitis per viam, /attendite, et videte /si est dolor sicut dolor meus " (traduction de la Bible de Jérusalem : «Vous tous qui passez par le chemin, regardez et voyez s'il est une douleur pareille à la douleur qui me tourmente »). Il est possible que ce poème ait été diffusé au moment liturgique de la semaine sainte où on lisait à l'office ce passage biblique. Voir F-B, éd. cit., t. 1, p. $257-258$.

37. C'est une suite de deux octosyllabes et d'un quadrisyllabe, avec la particularité que la rime du quadrisyllabe ne reprend pas celle des deux vers précédents, mais introduit une nouvelle rime, qui sera reprise par le tercet suivant, et ainsi de suite. Sur cette forme d'enchaînement continu et boiteux, voir Michel Zink, «Rythmes de la conscience. Le noué et le lâche des strophes 
médiévales ", dans La conscience de soi de la poésie : poésie et rhétorique, dir. Yves Bonnefoy, Paris, Lachenal \& Ritter, 1997, p. 58-68, en particulier p. 63-64.

38. Rutebeuf, Complainte de Guillaume, v. 8-13, dans F-B, éd. cit., t. 1, p. 258.

39. Boèce, La Consolation de Philosophie, livre 3, prose 5, éd.Claudio Moreschini, trad. Éric Vanpeteghem, Paris, Librairie Générale Français, coll. «Lettres gothiques», 2008, p. 156 (traduction: "Or quel fléau réussit mieux à nuire qu'un ennemi intime?»). La sentence, que traduira Rutebeuf dans une de ses autres pièces: "Et por ce nous dit ci la lettre : "Nule dolor n'est plus fervant /Qu'ele est de l'anemi servant" » (Des règles, v. 178-180, dans F-B, éd. cit., t. 1, p. 276), figure également chez Guillaume de Saint-Amour, attribuée de façon erronée à saint Grégoire dans le De periculis (chapitre, 3, éd. cit., p. 62) puis comme étant de son vrai auteur dans les Collectiones (voir F-B, éd. cit., t.1, p. 276). Si l'ouvrage du régent déchu est sans doute à l'origine immédiate de cette référence, rien n'oblige à refuser au ménestrel une connaissance plus directe de la Consolation ni à " penser que des maîtres universitaires ne sont pas loin » (Denis Huë et Hélène Gallé, Rutebeuf, op. cit., p. 75), ne fût-ce qu'eu égard à la vaste diffusion du texte au Moyen Âge, y compris en français (voir Jean-Yves Tilliette, "Introduction ", dans Boèce, La Consolation de Philosophie, éd.cit., p. 22-27; Antoine Thomas et Mario Roques, "Traductions françaises de la Consolatio Philosophiae de Boèce ", dans Histoire littéraire de la France, t. 23, Suite du quatorzième siècle, Paris, Imprimerie nationale, 1938, p. 419 - 488).

40. Un emploi métaphorique du verbe emprisoner renchérit sur la mise en parallèle du banni avec le prisonnier de Théodoric: "Et s'ont emprisoné mon pere/Dedenz sa terre", Rutebeuf, Complainte de Guillaume, v. 33 - 34, dans F-B, éd. cit., t. 1, p. 259.

41. Ibid., v. 108 - 110, dans F-B, éd. cit., t. 1, p. 263.

42. Comme intertexte lié à cette figure, Denis Hüe attire l'attention sur une lettre fictive de l'époque que l'Université aurait envoyée au roi. Son auteur anonyme y a inséré, pour évoquer les malheurs de Guillaume, quelques vers tirés de la Consolation (livre 1, mètre 5, éd. cit., p. 70-73) déplorant les agitations de Fortune. Ce qui prouverait que « le travail de Rutebeuf s'inscrit dans une démarche commune » coordonnée par son camp (Denis Hüe et Hélène Gallé, Rutebeuf, op. cit., p. 72 ; voir aussi F-B, éd. cit., t. 1, p. 256-257). Nous signalons toutefois que l'emploi décoratif qu'en a fait le pseudo-épistolaire n'est pas le même que celui de Rutebeuf qui, comme nous le verrons, apporte une distorsion à l'idée originale.

43. Voir Emmanuelle Métry, «Fortuna et Philosophia, une alliance inattendue. Quelques remarques sur le rôle de la Fortune dans la Consolation de philosophie de Boèce ", dans La Fortune : thèmes, représentations, discours, dir. Yasmina Foehr-Janssens et Emmanuelle Métry, Genève, Droz, 2003 , p. $59-70$.

44. Rutebeuf, Complainte de Guillaume, v. 171 - 173, dans F-B, éd. cit., t. 1, p. 265.

45. Jean-Yves Tilliette, «Introduction », dans Boèce, La Consolation de Philosophie, éd. cit., p. 36.

46. Certains traducteurs en ancien français de la Consolation rendent par complainte le latin querimonia pour désigner le chant initial où est évoquée la perfidie de Fortune. On lit «ma plorable complainte » chez un anonyme bourguignon au premier tiers du XIII ${ }^{\mathrm{e}}$ siècle, comme plus tard chez Jean de Meun (Antoine Thomas et Mario Roques, «Traductions françaises de la Consolatio », art. cit., p. 424 ; Venceslas Louis Dedeck-Héry, «Boethius' De Consolatione by Jean de Meun », Mediaeval Studies, vol. 14, 1952, p. 165 - 275, cit. p. 172).

47. Rutebeuf assure que «Diex, qui est et qui sera /s'il veut, en pou d'eure fera/Cest bruit remaindre » (Complainte de Guillaume, v. 159 - 161, dans F-B, éd. cit., t.1, p. 265). Mais les vers 171-173, cités ci-dessus, obscurcissent vite cette espérance, dont l'incertitude est paradoxalement intensifiée par l'adverbe « donc».

48. Le toponyme équivoque du refuge de Guillaume est suggestif : «Or est en son païs reclus, /A Saint Amor » (ibid., v. 66 - 67, dans F-B, éd. cit., t. 1, p. 261).

49. Ibid., v. 38, dans F-B, éd. cit., t. 1, p. 259. 
50. Emmanuelle Poulain-Gautret fait remarquer que la prétérition est utilisée plus d'une fois dans la Complainte de Guillaume (v. 68-70, v. 103 - 106, v. $117-120$ et v. 168, dans F-B, éd. cit., t. 1, p. 261, p. 263 et p. 265). Nous n'adhérons toutefois pas à son opinion selon laquelle ce procédé « contribue en effet à obscurcir encore l'identité et le rôle du locuteur » («"Mout a entre faire et dire" ", art. cit., p. 694).

51. F-B, éd. cit., t. 1, p. 34 - 35. Suivant cette idée, Denis Hüe considère qu' « anonyme, il ne risque pas grand-chose » malgré sa volonté de martyre affichée à la fin du Dit de Guillaume (Denis Hüe et Hélène Gallé, Rutebeuf, op. cit., p. 68). Signalons seulement qu'il n'est pas sûr que l'anonymat ait été un bon couvert devant les oppresseurs, a fortiori si l'on pense que « rien ne s'oppose à ce que Rutebeuf ait non seulement composé mais aussi dit ses textes » (ibid., p. 40), c'est-à-dire en face-àface avec le public.

52. De fait, selon la chronologie d'Edmond Faral, la plus ancienne signature de Rutebeuf se trouve dans une pièce encore pamphlétaire, le Dit d'Hypocrisie écrit en 1261 (v. 42 - 53 et v. 172, dans F-B, éd., cit., t. 1, p. 289 et p. 293 ; voir ibid., t. 1, p. 287). Le même philologue considère aussi que le poète a pu recevoir son nom, sans doute calqué sur le latin rudis bos, "dans la société de compagnons d'école » (ibid., t. 1, p. 34). Ces hypothèses peuvent corroborer la nôtre.

53. Rutebeuf, Dit des règles, v. 1 - 5, dans F-B, éd. cit., t. 1, p. 269.

54. Michel Foucault, «Qu'est-ce qu'un auteur? » [1969], dans Dits et écrits 1954-1988, t. I, 1954-1969, Paris, Gallimard, 1994, p. 789-821, cit. p. 799.

\section{AUTEUR}

\section{SUNG - WOOK MOON}

Sorbonne Université - Faculté des Lettres 